\title{
Criminologie
}

\section{La prédiction du comportement violent : exercice nécessaire et délicat}

\section{Bruno Marceau}

Volume 19, numéro 2, 1986

La prédiction de la carrière criminelle

URI : https://id.erudit.org/iderudit/017242ar

DOI : https://doi.org/10.7202/017242ar

Aller au sommaire du numéro

Éditeur(s)

Les Presses de l'Université de Montréal

ISSN

0316-0041 (imprimé)

1492-1367 (numérique)

Découvrir la revue

Citer cet article

Marceau, B. (1986). La prédiction du comportement violent : exercice nécessaire et délicat. Criminologie, 19(2), 101-115.

https://doi.org/10.7202/017242ar
Résumé de l'article

Discussions of dangerousness generally tend to be concentrated on the danger of violence in particular. The effective prediction of violent behaviour, if, indeed, it is the best guage of the objectivity of our knowledge of this phenomenon, is nonetheless an extremely difficult task. This article gives us an idea of some of the most current obstacles that confront the undertaking of prediction. Some additional thoughts follow, based on the author's earlier work on the subject. 


\title{
LA PRÉDICTION DU COMPORTEMENT VIOLENT :
} EXERCICE NÉCESSAIRE ET DÉLICAT Bruno Marceau*

\begin{abstract}
Discussions of dangerousness generally tend to be concentrated on the danger of violence in particular. The effective prediction of violent behaviour, if, indeed, it is the best guage of the objectivity of our knowledge of this phenomenon, is nonetheless an extremely difficult task. This article gives us an idea of some of the most current obstacles that confront the undertaking of prediction. Some additional thoughts follow, based on the author's earlier work on the subject.
\end{abstract}

\section{INTRODUCTION}

$\mathrm{Au}$ cours des dernières décennies, le délinquant violent est devenu objet de recherche privilégié. Deux raisons, entre autres, expliquent cet intérêt. D'une part, l'on commençait à reconnaître le caractère hétérogène de la délinquance; à phénomènes distincts devaient correspondre des causes distinctes. D'autre part, de tous les comportements délinquants, le comportement violent intentionnel est facilement perçu comme celui qui porte l'atteinte la plus profonde à certaines des valeurs les plus chères à notre société. Le délinquant violent, à bien des égards, en est venu à être perçu comme l'archétype du danger qui nous guette tous et la science, sous la pression du public et des instances publiques, consacre des efforts considérables à son identification dans l'espoir de le neutraliser, sinon de le traiter.

Récemment, nous avions l'occasion de procéder à un bilan des recherches scientifiques sur le sujet (Marceau, 1985). Nous devions alors constater l'apparente impossibilité de réduire le phénomène de la dangerosité de violence à une classe particulière d'individus aux caractéristiques plus ou moins identiques. Malgré les nombreux travaux qui leur ont été consacrés jusqu'ici,

* L'auteur est présentement chercheur à l'emploi de la Division de la recherche du ministère du Solliciteur général du Canada. Toutefois, les opinions exprimées dans cet article sont celles de l'auteur et ne sauraient être interprétées comme reflétant la position de son employeur. 
on ne peut conclure que la science se soit montrée, à ce jour, capable de décrire ces individus de manière intelligible, c'est-àdire en spécifiant chez eux des uniformités ou régularités susceptibles d'être vérifiées intersubjectivement.

Avec le développement de méthodes statistiques avancées et le recours aux ressources de l'informatique, on espérait réussir là où le jugement clinique et la théorie avaient échoué, mais peu importe la méthode utilisée, actuarielle, clinique ou mixte, on a dû généralement s'admettre impuissant à reconnaître les caractéristiques de la dangerosité et à prédire, individuellement et à long terme, le comportement violent, sans pour cela identifier à tort, comme dangereux, un grand nombre d'individus non violents.

Lorsque, à la suite de ces travaux, on pouvait déceler une quelconque relation statistique entre la violence et certaines caractéristiques individuelles susceptibles d'expliquer, mathématiquement, une partie de la variance dans le critère, on devait concéder que, bien que ces relations aient une certaine portée empirique, elles ne permettaient pas de prédire avec suffisamment de précision l'occurrence de nouveaux comportements violents. D'ailleurs, même si la variance expliquée devait être interprétée comme une indication de notre degré de connaissance du phénomène étudié, nous devrions admettre qu'en ce domaine, compte tenu de la faible proportion de cette variance expliquée, notre connaissance est largement dépassée par notre ignorance. Cela est particulièrement évident lorsque l'on en est réduit à avouer que le meilleur prédicteur de violence demeure la violence antérieure.

Il peut bien sûr sembler pas trop radical de réduire l'évaluation de notre connaissance d'un phénomène à notre aptitude à en prédire l'occurrence avec exactitude. Force est cependant de concéder d'une part que cette aptitude constitue l'indice le plus probant de l'objectivité de cette connaissance et d'autre part que c'est précisément sur cette capacité que l'on cherche à s'appuyer, sur le plan moral, pour justifier l'imposition de mesures préventives à l'égard d'individus que l'on qualifie de dangereux.

Il conviendrait donc de nous pencher un moment sur la nature des problèmes associés à la prédiction de la violence afin d'estimer, si possible, dans quelle mesure ceux-ci pourraient, éventuellement, être solutionnés. 


\section{QUELQUES PROBLĖMES COURANTS}

Ces problèmes de prédiction peuvent être classés sous trois rubriques bien que celles-ci ne soient pas mutuellement exclusives et qu'elles aient des effets interactifs qui rendent d'autant plus ardue la recherche de solutions globales. Ces trois ordres de problèmes peuvent être ramenés à l'absence apparente de consensus sur la définition de critères valables de dangerosité et de violence, à l'impossibilité pratique de vérifier la justesse des prédictions de violence, et enfin à la rareté relative du ou des types de comportements que l'on cherche à prédire.

\subsection{L'IMPRÉCISION DU CONCEPT}

Depuis un certain temps, on s'accorde généralement pour affirmer que plusieurs des problèmes reliés à l'évaluation du caractère dangereux d'un individu sont fonction du contexte assez particulier dans lequel une telle évaluation doit généralement être effectuée.

Plusieurs lois confient à des «experts» la tâche d'identifier les individus dangereux qui devront faire l'objet de mesures préventives spéciales. Or, certaines de ces législations négligent de préciser la nature du danger auxquelles elles font allusion, et même lorsqu'elles restreignent la définition de la dangerosité à une «propension à la violence», cela, il convient de l'avouer avec Diamond (1974), n'est pas d'un grand secours puisque la violence constitue elle-même un concept extrêmement difficile à définir.

En définitive, lorsque l'on demande à un expert de déterminer si un individu est dangereux, cet évaluateur est laissé à lui-même. Il devra donc construire lui-même son propre critère. Il ne faudrait pas s'étonner, dans ces circonstances, si l'expert présentait une évaluation qui reflète avant tout ses perspectives personnelles sur le délinquant, les finalités du système pénal, et son rôle propre à l'intérieur de ce système (voir Poupart et al., 1982). On pourrait difficilement le blâmer d'agir ainsi.

Or, la plupart des législations occidentales sur les délinquants dangereux, et la nôtre ne fait pas exception, confient le rôle d'expert aux psychiatres. Ceux-ci ont une formation clinique, leur vocation est de diagnostiquer et de traiter, d'où cette tendance à considérer la dangerosité comme un trait de personnalité, une pathologie. La violence devient elle-même 
une notion abstraite, un attribut de la personnalité qui n'a pas besoin d'être actualisé par un comportement, un acte de violence, pour pouvoir à tout le moins être présumé. D'ailleurs, dans un domaine comme celui de la santé, on présumera la maladie plutôt que la santé. On ne prend pas de chance avec la possibilité d'un cancer; pourquoi en prendrait-on avec la possibilité d'une «dangerosité», surtout lorsque le sujet a déjà démontré une telle dangerosité, par le passé, en commettant un ou des actes de violence.

Cette évaluation de la dangerosité constitue donc un diagnostic de l'état actuel d'un patient, et, éventuellement, un pronostic de son état futur. Elle ne constitue aucunement une prédiction de son comportement, si ce n'est par une extrapolation intuitive, dont le résultat demeure secondaire et n'est pas susceptible de remettre en cause la validité du diagnostic initial.

Les facteurs de personnalité sont évidemment ceux qui retiennent l'attention, au détriment des facteurs externes ou de facteurs situationnels. On ne veut pas nier ouvertement l'importance de ces derniers en tant que déclencheurs du comportement violent, mais on semble présumer que ces situations particulières ne constituent que des occasions, impossibles à déterminer et laissées au hasard, où le sujet agira violemment, parce qu'il est, fondamentalement, dangereux, violent.

Cette logique constitue certes une excuse facile pour contester l'utilisation du comportement violent ultérieur du sujet comme critère objectif de la validité des pronostics de dangerosité. Elle ne peut cependant expliquer que l'on continue à diagnostiquer la dangerosité, malgré l'accumulation de travaux indiquant qu'il n'existe aucune nomenclature psychiatrique ou psychologique appropriée pour décrire l'état dangereux comme un trait individuel spécifique. Comme Schlesinger (1978) le fait remarquer, on n'a pas encore réussi à établir que la dangerosité puisse exister séparément des actes par lesquels on l'identifie, ou qu'elle puisse constituer une entité, un facteur, ou un trait propre. Certains travaux tendent même à indiquer que la présence d'actes de violence antérieurs chez un sujet, à défaut de constituer un bon prédicteur de violence future, constitue par contre un excellent prédicteur du diagnostic et du pronostic de dangerosité.

Pourtant, le clinicien ne semble pas faire un recours conscient à la violence antérieure pour prédire la violence future. 
Il s'agirait plutôt, selon Pfohl (1978), d'un phénomène de "reconstruction sociale», où toute information, même redondante, est retenue, au détriment d'éléments parfois plus importants qui viendraient contrecarrer l'interprétation initialement retenue. Ce processus augmente bien sûr la confiance du clinicien, mais n'ajoute pas à la validité de son pronostic, au contraire.

Il est important de souligner encore que ce processus est essentiellement rétrospectif. Le comportement antérieur du sujet est réinterprété en fonction de ses caractéristiques personnelles actuelles et de certaines informations antérieures qui cadrent avec les conceptions du clinicien. De là, on présumera successivement : que le sujet était dangereux, ce qui semble acquis; qu'il est encore dangereux, puisque ses caractéristiques sont «demeurées» les mêmes; et qu'il sera dangereux pour une période indéterminée, c'est-à-dire tant que certains traits, présumés stables et arbitrairement associés à son comportement antérieur, ne seront pas affectés par le traitement... ou le passage du temps.

Inutile de dire que cette interprétation a posteriori, s'appuyant sur la «découverte» de traits actuels, dont la stabilité est présumée pour simultanément expliquer le comportement antérieur et prédire le comportement futur, demeure strictement spéculative.

Mais ce n'est pas tout.

\subsection{DES PRÉDICTIONS INVÉRIFIABLES}

Non seulement l'évaluateur est-il difficilement en position de vérifier la justesse de son interprétation rétrospective, mais habituellement, il ne pourra non plus s'assurer de la validité de ses "prédictions", étant donné que celles-ci, plus souvent qu'autrement, si elles penchent du côté de la dangerosité, seront suivies de mesures restrictives, avec ou sans traitement.

En fait, il n'est pas tout à fait juste de dire que les prédictions relatives à la dangerosité sont toutes invérifiables. Il faut ici distinguer entre deux types d'erreurs, dont l'un se prête rarement à la vérification, mais dont l'autre peut aisément être constaté. 
Le premier type d'erreurs consiste à identifier comme dangereux un individu, alors que cet individu, par la suite, ne manifesterait aucune violence comportementale : c'est le faux positif. On pourra rarement constater ce type d'erreurs, puisque le sujet sera alors habituellement interné.

Si, par ailleurs, le sujet considéré dangereux par l'expert était néanmoins libéré, il sera toujours possible de trouver une excuse plausible à l'erreur de type «faux positif» en s'en prenant encore une fois à la définition d'un critère que l'on qualifiera d'invalide, d'arbitraire, ou d'irréaliste. Peut-être le sujet a-t-il commis des actes de violence que les instances officielles n'ont pu détecter, ou peut-être la prédiction se serait-elle réalisée si l'on avait considéré une période de risque un peu plus longue...

Le second type d'erreurs consiste à qualifier de nondangereux un individu qui, dans un avenir plus ou moins immédiat, fera mentir l'évaluateur en commettant un acte de violence : c'est le faux négatif. Si le phénomène du chiffre noir nuit, encore ici, à la détection de l'ensemble des erreurs de ce type, il reste que ces erreurs sont beaucoup plus faciles à détecter, pour la simple raison que le sujet est habituellement libéré après l'évaluation.

S'il est vrai que c'est par la constatation de nos erreurs que l'on apprend et que l'on peut corriger notre action, on ne peut que déplorer le fait que les experts ne puissent apprendre que par leurs erreurs de ce deuxième type. De cette manière, la correction qui paraît s'imposer pointe toujours dans la direction d'une plus grande sécurité, par la diminution des erreurs de type faux négatif. Cette correction risque fort, à son tour, d'augmenter le nombre de faux positifs, puisque deviendront des «positifs» les individus qui présentaient des caractéristiques similaires aux faux négatifs constatés.

Cette dernière constatation nous amène tout naturellement à la discussion de cet autre problème chronique qui affecte l'exactitude des évaluations de dangerosité et la prédiction du comportement violent.

\subsection{LE PROBLÈME DES EFFECTIFS}

Malgré qu'elle n'en soit pas moins déplorable, la violence, statistiquement parlant, demeure un comportement rare. Non 
seulement est-elle rare dans une population générale, mais elle l'est encore, bien que sans doute à un degré moindre, dans des populations délinquantes ou criminelles comme le montrent, par exemple, les études de Wenk et al. (1972) ou de Schlesinger (1978), pour ne citer que celles-là.

Une foule de commentateurs ont eu l'occasion d'attirer notre attention sur les conséquences de la rareté des comportements violents, lorsqu'on cherche à en prédire l'occurrence sans la présumer trop souvent à tort (Cocozza et Steadman, 1976; Ennis et Emery, 1978; Ennis et Litwack, 1974; Livermore et al., 1968; Megargee, 1976; Monahan, 1975, 1981; Rubin, 1972; Steadman, 1976; Von Hirsh, 1972; Wenk et al., 1972). Et même si ce problème touche l'ensemble des méthodes de prédiction, il y a lieu de croire que l'évaluation clinique, fortement personnalisée, y soit encore plus vulnérable, en négligeant certaines réalités statistiques fondamentales (Monahan, 1981; Tverski et Kahneman, 1974).

C'est sans doute à Meehl et Rosen que revient le mérite d'avoir exposé le plus clairement, il y a déjà plus de trente ans les problèmes soulevés par un faible effectif (Meehl et Rosen, 1955; Rosen, 1954). Les travaux de ces auteurs ne portent pas sur l'estimation de la dangerosité ou la prédiction de la violence, mais les problèmes qui y sont présentés sont semblables à ceux que doivent affronter dans ces cas cliniciens, juges ou organismes de libération conditionnelle. La discussion théorique présentée par Rosen prend comme exemple le comportement le plus rare, selon lui, que les cliniciens soient appelés régulièrement à prédire : le suicide.

D'après les études existantes sur la question, Rosen estime que le taux de suicide dans une population psychiatrique est d'environ 3,3 sur mille. Ainsi, sur 12000 patients hypothétiques, on pourrait s'attendre à 40 suicides. En prédisant, sans l'aide d'aucun test et sans chercher à faire de distinctions, qu'aucun patient ne se suiciderait, on ne risquerait de se tromper que dans environ un tiers de $1 \%$ des cas; en d'autres termes, une telle prédiction serait juste dans environ $99,67 \%$ de ces cas. Rosen imagine alors la construction et l'utilisation d'un indice servant à prédire le suicide. Un tel indice, selon lui, ne pourrait identifier correctement une forte proportion de vrais positifs (sujets réellement suicidaires), qu'au prix d'un grand nombre de faux positifs (sujets identifiés comme suicidaires mais qui ne le sont 
pas en réalité). Si l'on cherchait ensuite à réduire le nombre de faux positifs, graduellement on ne pourrait plus identifier qu'une faible partie des vrais positifs, et encore là, les faux positifs resteraient plus nombreux que les vrais positifs.

Si l'instrument utilisé permettait par exemple de repérer correctement $75 \%$ des patients suicidaires et une proportion identique des non-suicidaires, on pourrait ainsi identifier correctement 30 des $\mathbf{4 0}$ sujets du premier groupe, mais en leur assimilant faussement 2990 sujets du second groupe. La réduction du nombre de faux positifs de $25 \%$ à $10 \%$ abaisserait à 1196 le nombre de sujets non suicidaires incorrectement prédits comme suicidaires, alors qu'on ne pourrait plus identifier que 24 des 40 patients réellement suicidaires. En voulant encore réduire la proportion de faux positifs à $0,5 \%$, ce qui en laisserait encore 60 , on ne pourrait plus repérer qu'un seul des $\mathbf{4 0}$ patients suicidaires.

Comme on peut facilement le constater, même si un instrument a une valeur intrinsèque, c'est-à-dire même s'il distingue généralement bien les gens chez qui un trait est présent de ceux qui en sont exempts, il demeure que la distribution de ce trait dans la population concernée peut influencer son utilité pratique. Meehl et Rosen (1955) ont démontré que lorsqu'il est appliqué dans une population où le taux de base du trait s'éloigne considérablement de $50 \%$, un instrument de classification ou de prédiction possédant une puissance discriminante raisonnable peut néanmoins produire plus d'erreurs de diagnostic que si l'on s'était fié uniquement au taux de base pour déclarer, selon le cas, qu'aucun des sujets de la population ne possédait ce trait, ou qu'au contraire, le trait était présent chez tous les individus.

Ainsi, lorsqu'une caractéristique est rare dans une population, un instrument qui permet de repérer la plupart ou même la totalité des sujets qui possèdent cette caractéristique, tout en ne l'attribuant par erreur qu'à une faible proportion de sujets qui en sont en réalité exempts, peut malgré tout conduire à plus d'erreurs de diagnostic ou de pronostic, en chiffres absolus, que l'on en aurait faites en considéant tout simplement qu'aucun des sujets ne possédait ladite caractéristique. $\mathrm{La}$ "faible proportion» de sujets à qui l'on attribue faussement une caractéristique rare dans une population peut en effet représenter un grand nombre d'erreurs réelles.

Pour contrer au moins partiellement ces difficultés, Rosen (1954) suggérait, quant à lui, de concentrer les efforts de prédic- 
tion sur certains groupes particuliers de sujets où le taux de base du trait ou du comportement en question serait plus élevé. Cette stratégie, comme il le faisait remarquer, comporte certains aspects négatifs. D'une part, il demeurerait encore difficile pour certains comportements comme le suicide de trouver des groupes où le taux de base serait suffisamment élevé. D'autre part, on risquerait alors, en se concentrant sur certains sous-groupes, de négliger une partie importante des sujets qui manifesteraient effectivement le comportement que l'on cherche à prédire. Selon Von Hirsh (1972), ces mêmes difficultés se présentent lorsque l'on cherche à prédire le comportement violent. Il serait en effet difficile de trouver des sous-groupes d'individus où ce comportement est suffisamment fréquent, et ceux-ci ne seraient responsables que d'une faible proportion de l'ensemble de la violence; ainsi, même si l'on parvenait à identifier et à juguler les sujets effectivement violents de ces sous-groupes particuliers, cela contribuerait très peu à améliorer la sécurité publique.

Comme Von Hirsch le fait par ailleurs remarquer, on peut alors chercher à gonfler le taux de base en considérant, comme faisant partie du danger que l'on cherche à identifier et prévenir certaines infractions de moindre gravité ou certaines infractions non violentes. Il faudrait cependant alors s'interroger sur la nécessité ou la pertinence des mesures que nos prédictions pourraient sembler autoriser.

Qu'il s'agisse de la difficulté de s'entendre sur un critère approprié, de celle posée par la rareté du comportement violent, ou encore des difficultés attachées au contexte dans lequel on procède souvent à la prédiction, voilà autant d'embûches propres à décourager plus d'un chercheur.

Ces embûches sont, bien entendu, celles que rencontre plus particulièrement le chercheur praticien, dont les prédictions auront des conséquences immédiates.

Mais qu'en serait-il pour le chercheur qui, dégagé des contraintes pratiques que doit affronter l' «expert», n'aurait à répondre que devant la science?

\section{UNE ILLUSTRATION PERSONNELLE}

Il est possible en effet de chercher à recréer des circonstances idéales, exemptes autant que possible des problèmes 
susmentionnés. Dans ces circonstances, on serait libre d'opter pour un critère quelconque qui apparaît plus naturel que les définitions juridiques; on pourrait réajuster le taux de base pour qu'il se rapproche de $50 \%$, sans pour autant recourir à un élargissement inconsidéré du critère; et dans ces circonstances, enfin, la prédiction, éventuellement fondée sur une explication, ne comporterait de conséquences que pour la science elle-même.

C'est dans un tel cadre «idéal» que nous avons eu l'occasion d'effectuer nos travaux. Privilégié, nous l'étions également à deux autres points de vue, soit par l'accès aux techniques sophistiquées offertes par l'informatique, et par le volume d'information disponible sur un échantillon représentatif de 470 jeunes ayant comparu devant la Cour de bien-être social de Montréal il y a une douzaine d'années.

On trouvera le détail de ces travaux ailleurs (Marceau, 1981, 1985). Contentons-nous ici d'en signaler les grandes lignes. Nous avons d'abord établi notre propre critère de violence sous la forme d'un indice comportemental fondé sur les révélations faites par les sujets au cours d'entrevues en profondeur. À l'aide de cet indice, nous avons ensuite isolé deux classes de sujets, de taille relativement égale, qui nous assuraient du plus grand contraste possible entre sujets clairement violents et sujets tout à fait pacifiques. Une masse de données provenant des résultats des sujets sur cinq tests psychométriques fut ensuite soumise à l'analyse discriminante. Dans le but d'estimer la stabilité des distinctions obtenues à partir de ses premières analyses, le même type d'analyse fut répété en utilisant cette fois les résultats obtenus deux ans plus tard, par nos deux groupes de sujets, à l'Inventaire de Jesness qui apparaissait l'instrument susceptible de fournir la meilleure distinction entre les deux groupes.

Or, quels furent nos résultats? En apparence, ils étaient excellents. Comme il fallait s'y attendre dans ces circonstances, nous avons trouvé «quelque chose» susceptible d'une part d'expliquer mathématiquement un peu plus du tiers de la variance entre les groupes, et d'autre part de classer correctement de 75 à $80 \%$ des sujets à chacune des deux époques. Bien qu'en termes absolus, on puisse être déçu de ces premiers résultats, ils étaient comparables à ceux de la plupart des études sur la question. Et pourtant, ces résultats ne nous permettaient 
pas d'aller bien loin. Plusieurs raisons justifient notre prudence et de celles-ci, nous ne mentionnerons que les suivantes.

\subsection{LA DIFFICULTÉ D'ÉTABLIR UN LIEN DE CAUSALITÉ}

Une contrainte majeure affectait nos travaux, comme la plupart des recherches en ce domaine, en ce sens que notre exercice devait se limiter à une classification ou prédiction post facto, étant donné l'impossibilité de contrôler les effets possibles de l'intervention du tribunal auprès des sujets de notre échantillon.

Ceci pose un problème évident qu'il serait vain de chercher à dissimuler. Comme chacun le sait, l'établissement d'une relation de causalité exige l'antériorité de la cause sur les effets. Il est vrai que nos sujets, dont le comportement récent était marqué par des actes de violence, présentaient par la suite des caractéristiques individuelles qui les distinguaient, jusqu'à un certain point, des sujets qui n'avaient fait preuve d'aucune violence. De là à présenter ces caractéristiques individuelles comme des facteurs déterminants du comportement violent, plutôt que l'inverse, il y a un pas que nous refusons délibérément de franchir, sans nous être assuré, tout au moins, de la stabilité et, de ce fait, de l'existence de ces traits distinctifs antérieurement aux comportements différentiels. Or, cette stabilité, il nous demeurait interdit de la présumer d'autant plus que nous devions constater qu'en l'espace de deux ans, c'est-àdire lors de notre deuxième série d'analyses, les caractéristiques permettant la classification des sujets n'étaient apparemment plus les mêmes.

Le passage de la description à la théorie et de la classification à la prédiction véritable serait donc prématurée.

\subsection{LE PASSAGE DE L'IDÉAL À LA RÉALITÉ}

Abstraction faite de ce premier problème, il convient d'apporter une autre qualification quant à l'utilité réelle de notre instrument de prédiction.

En effet, notre étude opposait, de manière idéale et artificielle, deux groupes d'individus, de taille relativement égale. Il faut donc s'interroger sur la valeur éventuelle de notre instrument de classification dans des circonstances plus naturelles, où 
les distinctions sur le critère seraient moins tranchées, moins nettes, et où violents et non-violents ne se retrouveraient pas nécessairement en proportions égales. Nous avons exploré cette question après avoir établi à partir de données disponibles sur l'ensemble de l'échantillon d'où nos groupes avaient été sélectionnés, que la proportion de sujets violents ne pouvait y dépasser environ $25 \%$.

Postulant pareil taux de base, nous avons ensuite utilisé les formules recommandées par Meehl et Rosen, et elles-mêmes dérivées du théorème de Bayes, pour enfin constater que notre instrument ne produirait plus, dans un contexte réaliste, que des résultats à peine supérieurs à ceux que nous aurions obtenus en adoptant une règle de décision plus prudente, à savoir qu'aucun sujet de l'échantillon ne serait violent.

Nous avons par ailleurs procédé à un exercice plus spécifique en présumant que chacun des membres du groupe de nonviolents représentaient en fait trois sujets et que le point de décision sur notre fonction discriminante devait en conséquence être déplacé afin de ne considérer comme violents que le quart des sujets de l'échantillon qui se rapprochait le plus du pôle de violence. Bien qu'il soit difficile de justifier la pertinence de ce dernier exercice, une conclusion s'imposait. Cette conclusion, c'est que sur les sujets classés comme violents, plus de $30 \%$ ne l'étaient pas. Et encore faut-il demeurer conscient que cette conclusion témoigne elle-même de conditions idéalisées, artificielles, puisque nos sujets non violents étaient en fait les sujets les plus pacifiques de l'échantillon et ne pouvaient être considérés comme représentatifs de l'ensemble de ces délinquants qu'on ne peut qualifier de violents.

\subsection{UN EXERCICE TAUTOLOGIQUE?}

Les deux remarques précédentes ont déjà de quoi nous faire douter sérieusement de la valeur de nos résultats, mais un autre commentaire s'impose encore qui a trait, celui-là, à la nature de l'information qui nous avait permis, tant soit peu, d'établir des distinctions entre nos sujets violents et non violents.

En fait, ces distinctions étaient très plausibles, à tel point d'ailleurs qu'elles devaient nous inciter à soupçonner un certain degré de synonymie avec le critère. 
L'Inventaire de Jesness, que nous avons retenu, faute de mieux, dans nos dernières analyses, est un test objectif composé d'un certain nombre d'items, c'est-à-dire de questions formulées en des termes concrets et accessibles à une population d'adolescents. Le répondant s'y décrit en des termes à peine différents de ceux qu'il avait employés en racontant sa carrière délinquante. On peut d'ailleurs remarquer que Jesness lui-même, dans son manuel, recourt très peu à l'interprétation abstraite pour décrire la signification des différentes échelles de son test. Au contraire, il utilise abondamment les guillemets, le verbatim de ses questions et réponses.

L'interprétation des résultats, en définitive, dépend largement des conceptions personnelles de l'évaluateur. Notre point est le suivant. La description des distinctions entre nos deux groupes de sujets ne faisait qu'accumuler un certain nombre de caractéristiques plus ou moins équivalentes au critère. Cette accumulation de synonymes partiels, du mème ordre que le critère, pouvait difficilement mener à une explication de ce dernier. Cette simple retranscription ou reformulation du critère demeure largement un exercice tautologique. Nos groupes de sujets demeurent ainsi des groupes empiriques qu'il ne faut pas confondre avec des types d'une portée théorique quelconque.

Avant même de nous interroger sur nos capacités à expliquer, il convient donc de nous interroger sur nos aptitudes à décrire, c'est-à-dire à percevoir des caractéristiques de classes qui ne soient pas redondantes, et qui soient vraiment distinctes du critère initial. Ceci n'est d'ailleurs pas sans rappeler cette constatation que la violence, à défaut de permettre une prédiction adéquate de la violence ultérieure demeure souvent le meilleur prédicteur d'un diagnostic de dangerosité, peu importe les symptômes effectivement mentionnés pour justifier un tel diagnostic. Cette difficulté à décrire explique sans doute la pauvreté de nos explications, de notre connaissance du sujet. Le fossé entre l'empirisme et la théorie apparaît extrêmement difficile à combler.

\section{CONCLUSION}

Que ce soit à cause des multiples difficultés associées à la prédiction clinique du comportement violent individuel, ou que ce soit à cause de difficultés plus fondamentales à dépasser le 
stade de la description ou classification, la tendance de la littérature actuelle sur la question consiste à renoncer à la poursuite d'un modèle déterministe qui permettrait des prédictions individuelles assurées, pour viser plutôt à l'établissement de catégories de risques fondées sur un modèle strictement probabiliste plus ou moins exempts de contenu théorique.

Mais si l'utilisation du modèle probabiliste représente un progrès, c'est bien plus par son appel à la modestie du chercheur, que par le degré de connaissances qu'elle démontre. On peut en effet remarquer que les recherches sur l'évaluation des risques, qui utilisent un modèle probabiliste, sont elles-mêmes empreintes de difficultés comme en témoigne l'incapacité des études contemporaines à identifier des catégories d'individus où le risque de délits violents atteindrait un niveau de probabilité qui s'approche même de $50 \%$. En somme, l'utilisation d'un modèle probabiliste ne fait que confirmer l'extrême précarité de notre connaissance du délinquant violent ou dangereux, même s'il s'agissait là d'une connaissance purement a-théorique.

Enfin, il importe de demeurer conscient qu'un jugement probabiliste est essentiellement différent d'un jugement exprimé en termes déterministes, et notamment qu'il ne permet de prédire une occurrence particulière. Autrement dit, le fait qu'un individu fasse partie d'un groupe dont on sait que $40 \%$ des membres devraient faire preuve de violence, alors même qu'il s'agirait là du groupe où ce risque est le plus élevé, ne permettrait aucunement d'affirmer que cet individu commettra un acte de violence. La science semble donc encore loin de pouvoir assumer le rôle que nos institutions lui demandent de remplir.

\section{RÉFÉRENCES}

COCOZZA, J.J., H.J. STEADMAN (1976), "The Failure of Psychiatric Predictions of Dangerousness : Clear and Convincing Evidence», Rutgers Law Review, 29, 1084-1101.

DIAMOND, B.L. (1974), «The Psychiatric Prediction of Dangerousness", University of Pennsylvania Law Review, 123, 439-452.

ENNIS, B.J., R.D. EMERY (1978), The Rights of Mental Patients, New York, Avon.

ENNIS, B.J., T.R. LITWACK (1974), «Psychiatry and the Presumption of Expertise : Flipping Coins in the Courtroom», California Law Review, $62,693-752$. 
JESNESS, C.F. (1972), The Jesness Inventory Manual ( $2^{\mathrm{e}}$ éd. rév.), Palo Alto, Californie, Consulting Psychologists Press.

LIVERMORE, J.M., C.P. MALMQUIST, P.E. MEEHL (1968), "On the Justifications for Civil Commitment», University of Pennsylvania Law Review, 117, 75-96.

MARCEAU, B. (1985), le Délinquant violent devant la science et devant la loi. Thèse de doctorat inédite, Université de Montréal.

MARCEAU, B. (1981), «Quelques aspects du comportement violent chez les jeunes», Apprentissage et socialisation, 4, 90-100.

MEEHL, P.E., A. ROSEN (1955), «Antecedent Probability and the Efficiency of Psychometric Signs, Patterns, or Cutting Scores», Psychological Bulletin, 52, 194-216.

MEGARGEE, E.I. (1976), «The Prediction of Dangerous Behavior», Criminal Justice and Behavior, 3, 3-22.

MONAHAN, J. (1981), The Clinical Prediction of Violent Behavior, Rockville, Md., U.S. Department of Health and Human Services.

MONAHAN, J. (1975), "The Prediction of Violence», dans D. Chappell, J. Monahan (édit.), Violence and Criminal Justice, Lexington, Mass., D.C. Heath.

PFOHL, S.J. (1978), Predicting Dangerousness : the Social Construction of Psychiatric Reality, Lexington, Massachusetts, D.C. Heath.

POUPART, J., J. DOZOIS, M. LALONDE (1982), «L'expertise de la dangerosité», Criminologie, $15,\left(\mathbf{n}^{\circ} 2\right), 7-25$.

ROSEN, A. (1954), "Detection of Suicidal Patients : an Example of Some Limitations in the Prediction of Infrequent Events", Journal of Consulting Psychology, 18, 397-403.

RUBIN, B. (1972), «Prediction of Dangerousness in Mentally Ill Criminals», Archives of General Psychiatry, 27, 297-407.

SCHLESINGER, S.E. (1978), "The Prediction of Dangerousness in Juveniles : a Replication", Crime and Delinquency, 24, 40-48.

STEADMAN, H.J. (1976), «Predicting Dangerousness», dans D.J. Madden, J.R. Lion (édit.), Aggression and Violence, vol. 1, Rage-Hate-Assault and Other Forms of Violence, New York, Spectrum, pp. 53-69.

TVERSKI, A., D. KAHNEMAN (1974), «Judgment under Uncertainly; Heuristics and Biases", Science, 185, 1124-1131.

VON HIRSCH, A. (1972), «Prediction of Criminal Conduct and Preventive Confinement of Convicted Persons", Buffalo Law Review, 21, 717-758.

WENK, E.A., J.O. ROBISON, G.W. SMITH (1972), "Can Violence be Predicted?, Crime and Delinquency, 18, 393-402. 